\title{
Bronchitis obliterans after severe pneumonia in children: a review of 34 cases
}

\author{
Rui Zhou ${ }^{1}$ and Jihong Dai ${ }^{1}$ \\ ${ }^{1}$ Chongqing Medical University Affiliated Children's Hospital Department of Respiratory
}

September 25, 2021

\begin{abstract}
Abstract Background: This study aimed to describe the clinical characteristics, pathogeny and bronchoscopic findings of 34 children with bronchitis obliterans after severe pneumonia. Methods: Thirty-four children diagnosed with bronchitis obliterans were retrospectively studied at the Children's Hospital of Chongqing Medical University in China between January, 2014 and October, 2020. The clinical manifestations, pathogeny, lung computed tomography imaging and bronchoscopic findings of the 34 children were reported. Results: In children with bronchitis obliterans, repeated coughing, fever, wheezing, tachypnea, and lung rales were common clinical manifestations. Mycoplasma pneumoniae (M. pneumoniae) was the most common etiologic agent, followed by adenovirus. The CT scan images included uneven lung inflation(12/34), lung consolidation( 27/34), pulmonary atelectasis(23/34), and pleural effusion(22/34). All children underwent flexible bronchoscopy, and major types of airway findings were identified: mucosal congestion, luminal longitudinal folds, mucosal erosion or necrosis, hyperplastic changes, sputum emboli, bronchiectasis and obliterative-like lesions. Conclusion: In conclusion, severe M. pneumoniae pneumonia and adenovirus pneumonia appeared to have a higher risk of development of bronchitis obliterans. Bronchoscopy can be used as the main criterion for the diagnosis of bronchitis obliterans. Bronchoscopy interventional treatment is an effective measure to quickly recanalize the occluded lumen, but there is a risk of recurrence after treatment. Children with severe pneumonia should undergo regular follow-up and bronchoscopy to detect possible lumen occlusion in time.
\end{abstract}

Bronchitis obliterans after severe pneumonia in children: a review of 34 cases

Rui Zhou ${ }^{1,2}$, Jihong Dai ${ }^{1,2}$ MD, PhD

\section{Affiliations:}

Respiratory Department, Children's Hospital of Chongqing Medical University, Chongqing, China;

Ministry of Education Key Laboratory of Child Development and Disorders;

Corresponding author: Jihong Dai; Children's Hospital, Chongqing Medical University, 136, 2nd Road of Zhongshan, Yuzhong district, Chongqing, China;

Corresponding author: Jihong Dai E-mail:2200454523@qq.com

Rui Zhou E-mail: ruizhou420@163.com

\begin{abstract}
Background: This study aimed to describe the clinical characteristics, pathogeny and bronchoscopic findings of 34 children with bronchitis obliterans after severe pneumonia. Methods: Thirty-four children diagnosed with bronchitis obliterans were retrospectively studied at the Children's Hospital of Chongqing Medical University in China between January, 2014 and October, 2020. The clinical manifestations, pathogeny, lung computed tomography imaging and bronchoscopic findings of the 34 children were reported. Results:
\end{abstract}


In children with bronchitis obliterans, repeated coughing, fever, wheezing, tachypnea, and lung rales were common clinical manifestations. Mycoplasma pneumoniae (M. pneumoniae) was the most common etiologic agent, followed by adenovirus. The CT scan images included uneven lung inflation(12/34), lung consolidation( 27/34), pulmonary atelectasis(23/34), and pleural effusion(22/34). All children underwent flexible bronchoscopy, and major types of airway findings were identified: mucosal congestion, luminal longitudinal folds, mucosal erosion or necrosis, hyperplastic changes, sputum emboli, bronchiectasis and obliterative-like lesions. Conclusion: In conclusion, severe M. pneumoniae pneumonia and adenovirus pneumonia appeared to have a higher risk of development of bronchitis obliterans. Bronchoscopy can be used as the main criterion for the diagnosis of bronchitis obliterans. Bronchoscopy interventional treatment is an effective measure to quickly recanalize the occluded lumen, but there is a risk of recurrence after treatment. Children with severe pneumonia should undergo regular follow-up and bronchoscopy to detect possible lumen occlusion in time.

Key words: Bronchitis obliterans, pathogeny, bronchoscopy, pneumonia, children.

\section{Abbreviations:}

$\mathrm{BO}=$ Bronchiolitis Obliterans

SJS $=$ Stevens-Johnson syndrome

M. pneumonia $=$ Mycoplasma pneumoniae

$\mathrm{BALF}=$ bronchoalveolar lavage fluid

$\mathrm{PCR}=$ polymerase chain reaction

$\mathrm{CT}=$ computed tomography

Funding Sources: The authors state that this work has not received any funding.

Financial Disclosure: The authors have no financial relationships relevant to this article to disclose.

Conflict of Interest: The authors have no conflict of interest to disclose.

Contributor's Statement Page

Rui Zhou made substantial contributions to designing the study, collecting the data, analyzing the data, interpreting the results and preparing the manuscript. Jihong Dai assisted with interpreting the results and manuscript preparation. All authors reviewed and approved the final version of the manuscript.

\section{Hosted file}

manuscript.docx available at https://authorea.com/users/437074/articles/538967-bronchitisobliterans-after-severe-pneumonia-in-children-a-review-of-34-cases

\section{Hosted file}

Table 1.docx available at https://authorea.com/users/437074/articles/538967-bronchitisobliterans-after-severe-pneumonia-in-children-a-review-of-34-cases 


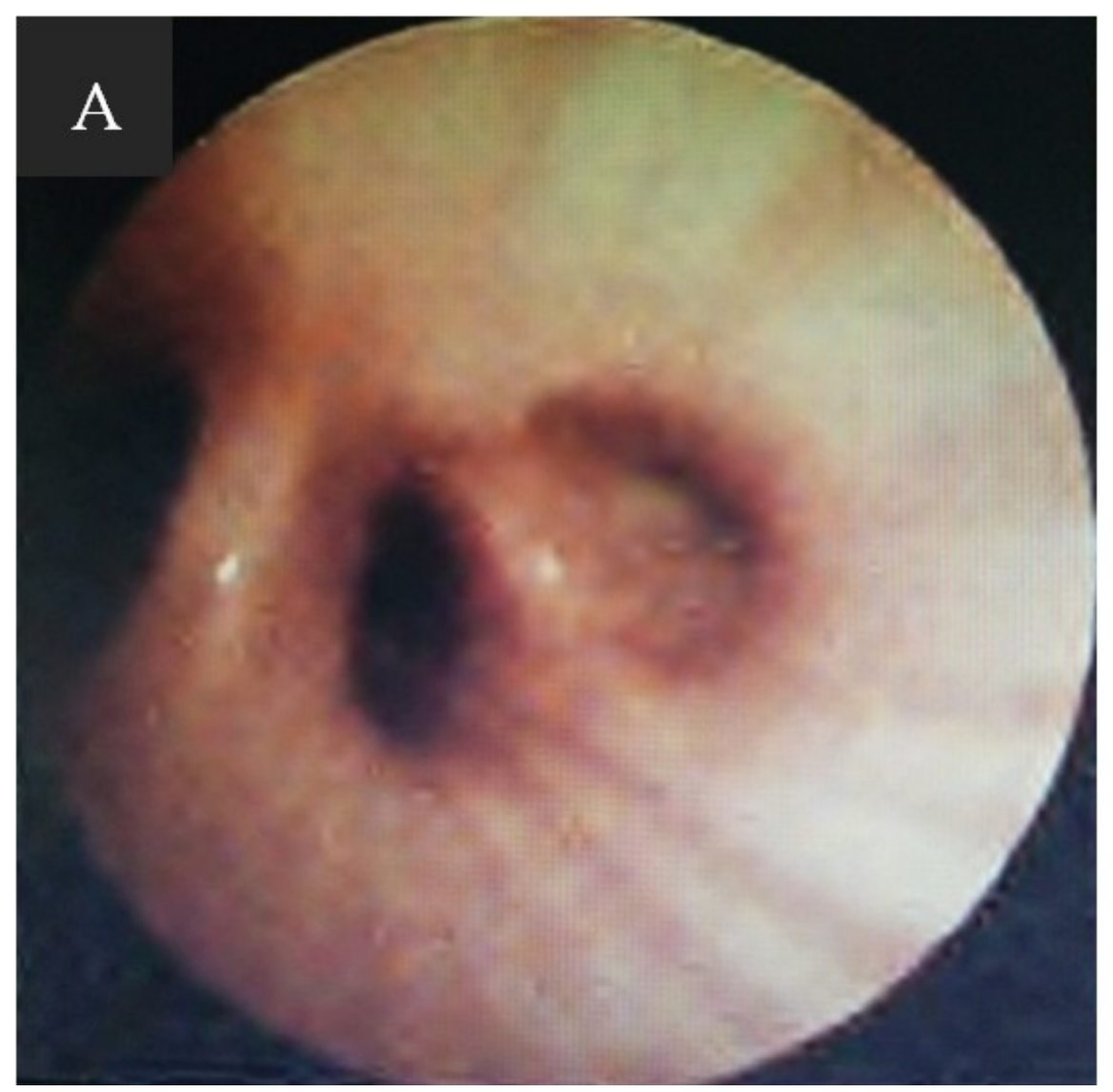




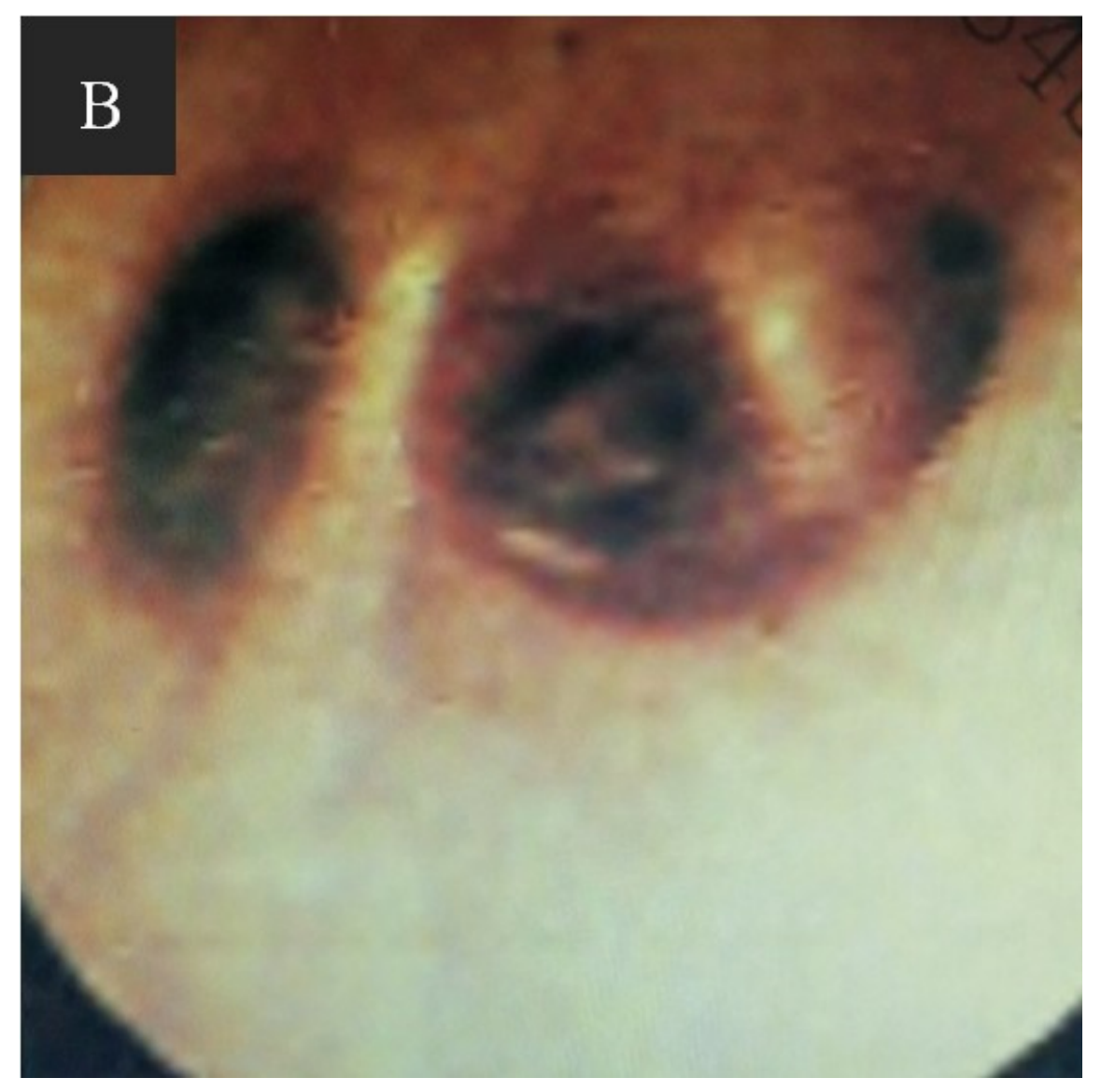




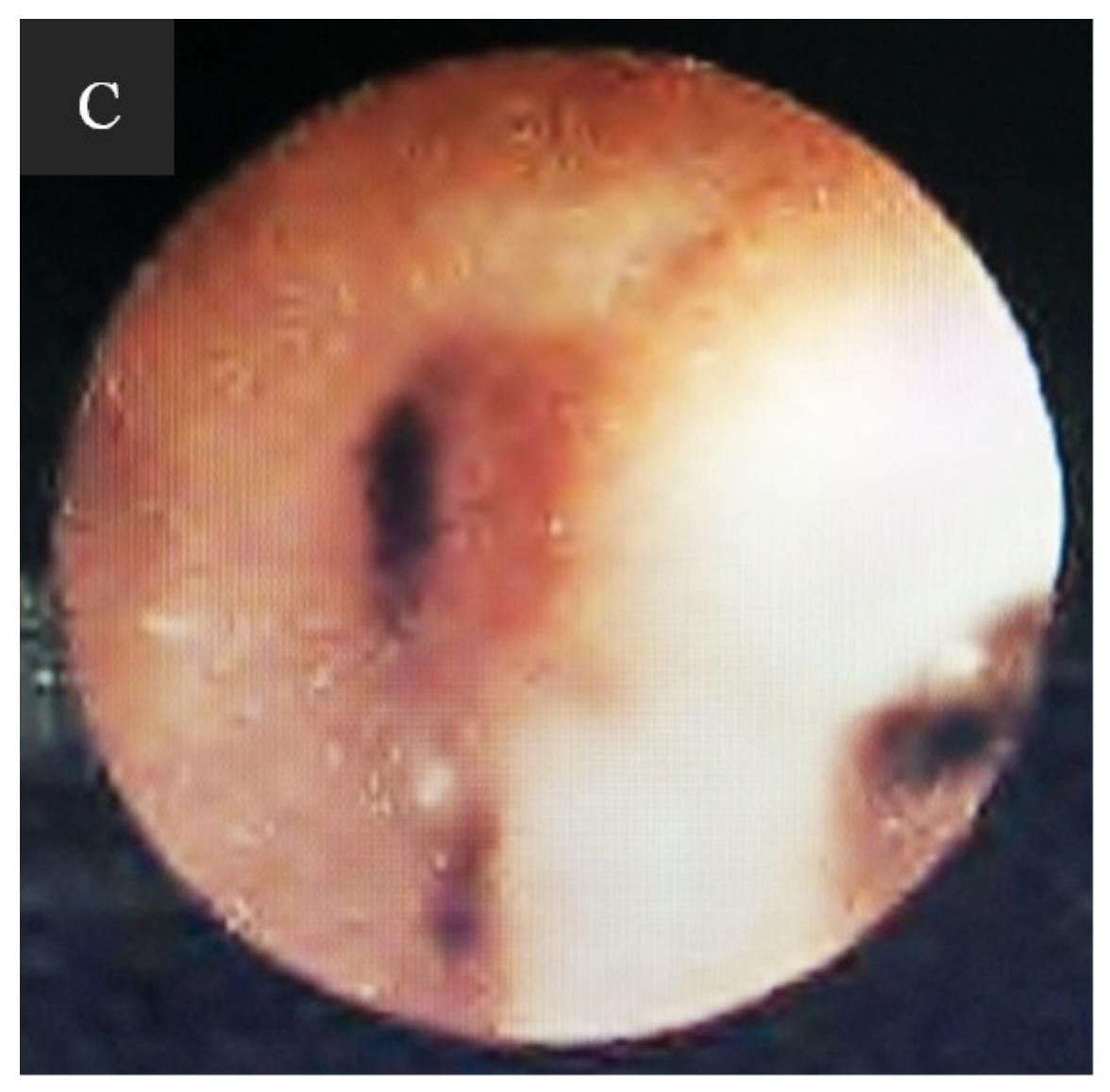




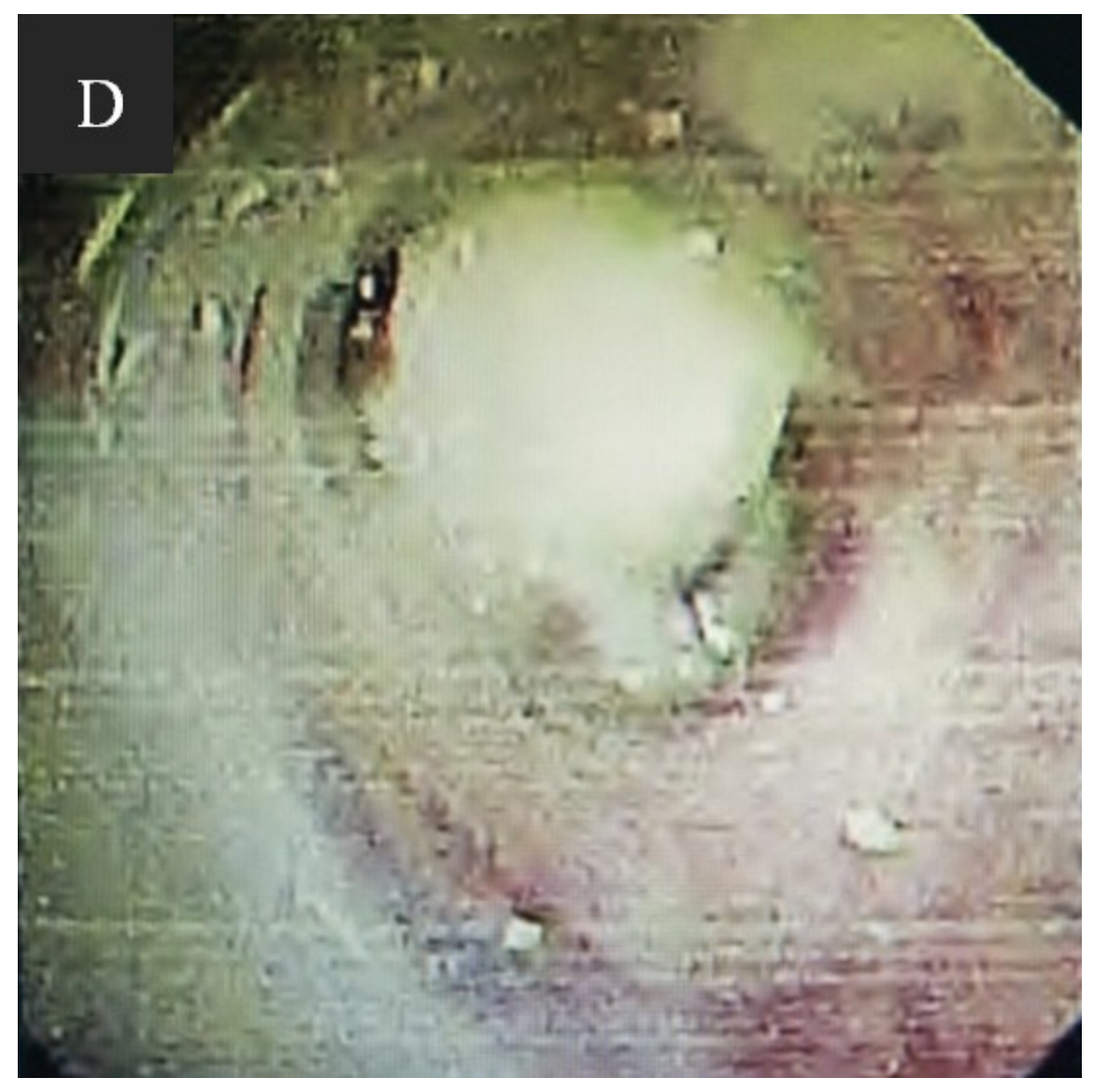




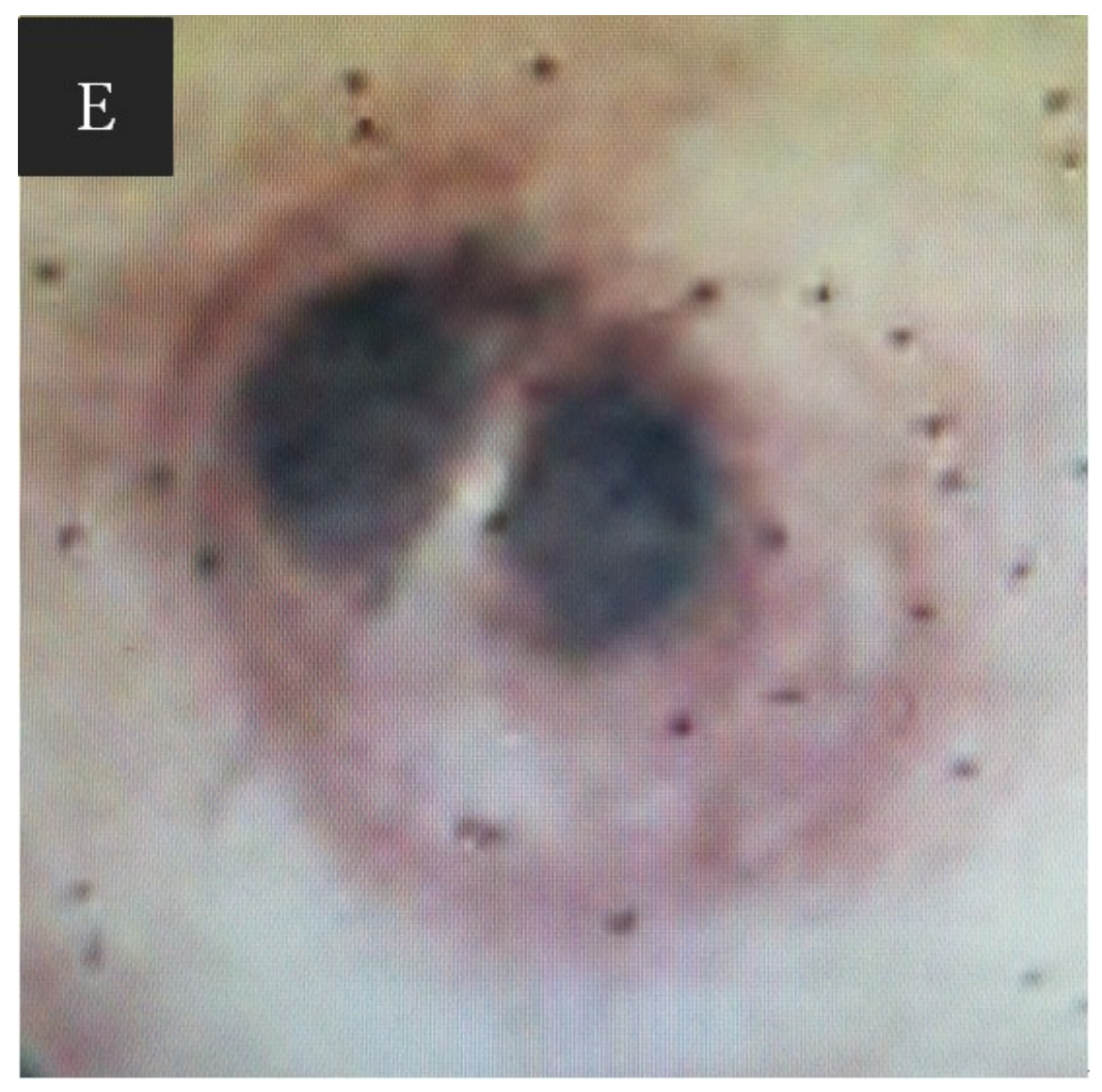




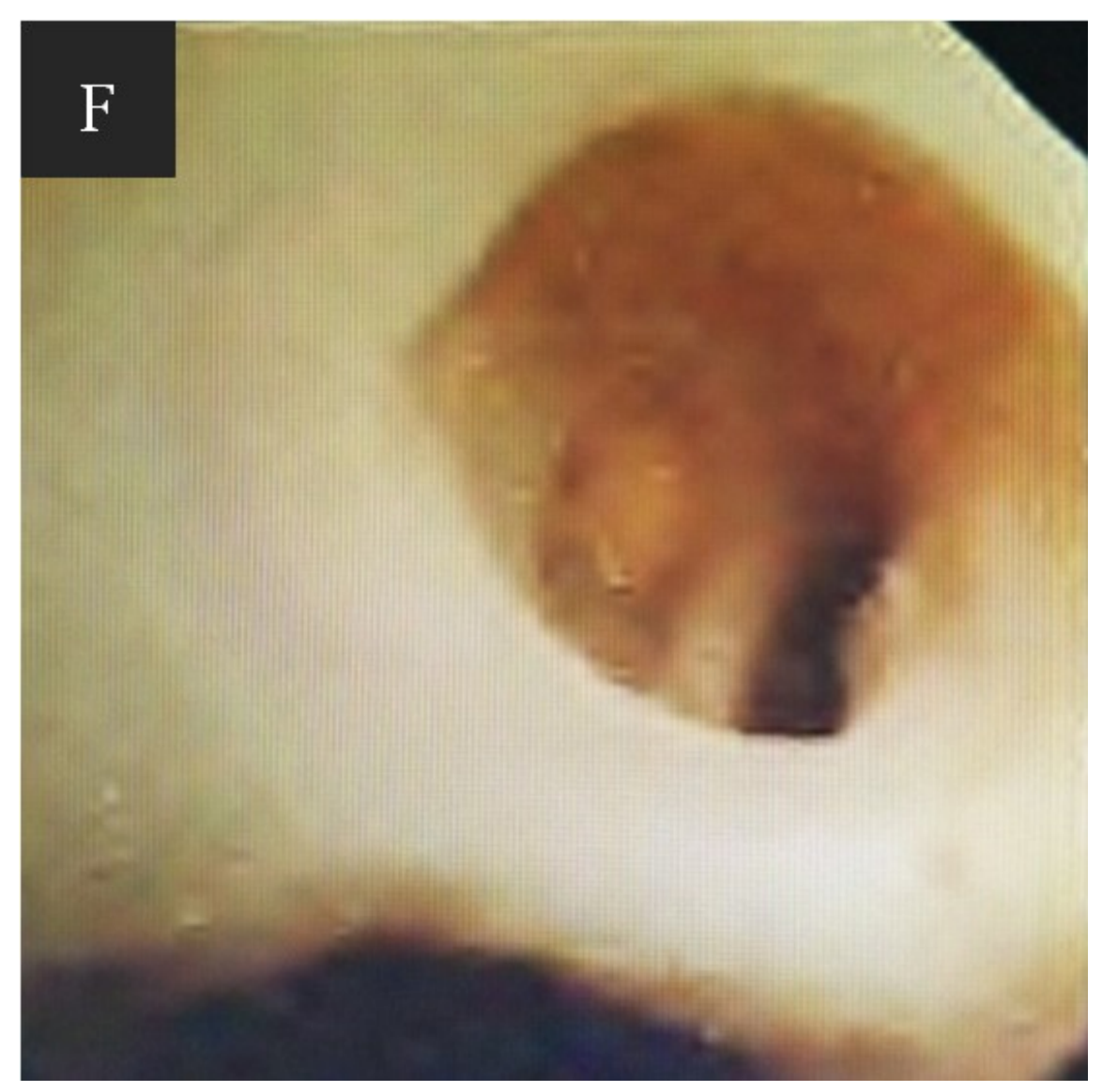

